\title{
Efeitos dos Fitoestrogênios sobre Alguns Parâmetros Clínicos e Laboratoriais no Climatério
}

\author{
Phytoestrogen Effects on Some Clinical and Laboratory Parameters in Climacterium
}

\begin{abstract}
Kyung Koo Han, José Maria Soares Júnior, Mauro Abi Haidar, Manoel João Batista C. Girão, Marcia Gaspar Nunes, Geraldo Rodrigues de Lima, Edmund C. Baracat
\end{abstract}

\section{RESUMO}

Objetivo: avaliar os efeitos estrogênicos de fitoestrogênios da soja em pacientes na pósmenopausa.

Métodos: foram avaliadas pacientes na pós-menopausa pré-selecionadas no Setor de Climatério do Departamento de Ginecologia da Universidade Federal de São Paulo- Escola Paulista de Medicina. As pacientes foram divididas em dois grupos de 40 cada: GI (isoflavona) - recebeu $100 \mathrm{mg}$ de isoflavona por dia, em cápsulas (divididas em 3 tomadas); e GCrt - recebeu placebo (controle). Na inclusão do trabalho, foram avaliadas por meio do índice de Kupperman, exame fisico e submetidas a dosagens bioquímicas de colesterol total e frações, triglicérides, estradiol, FSH e LH, além de avaliação ultra-sonográfica do eco endometrial. Para comparar os dois grupos antes e após o tratamento, utilizou-se o teste não paramétrico de Kruskal-Wallis para o índice de Kupperman, ao passo que os dados foram analisados pelo teste t para duas amostras independentes.

Resultados: no que se refere aos sintomas avaliados pelo índice de Kupperman, 80 \% das pacientes do GI (isoflavona) melhoraram, ao passo que no GCtr (controle) a melhora foi de apenas 12,5\%. Com relação aos níveis de colesterol total plasmático, das 35 pacientes do GI que apresentaram níveis superiores a $200 \mathrm{mg} / \mathrm{dL}$, ao passo que a queda registrada no GCtr foi em apenas 13 pacientes. Com relação ao índice de massa corpórea, houve redução no grupo GI (isoflavona), mantendo-se estável no GCtr (controle). Notamos também que a espessura endometrial e os niveis sérios de FSH não apresentaram variações significativas. Houve elevação dos valores de estrogênio após o tratamento com isoflavona.

Conclusão: concluímos que a isoflavona, na dose de $100 \mathrm{mg} /$ dia, pode ser utilizada como alternativa para atenuar os sintomas climatéricos. Comprovamos que houve redução dos níveis de colesterol total plasmático.

PALAVRAS-CHAVE: Fitoestrogênio. Climatério. Menopausa. Índice de Kupperman.

\section{Introdução}

A ação estrogênica e antiestrogênica de algumas substâncias derivadas de plantas é conhe-

Departamento de Ginecologia da Universidade Federal de São Paulo - Escola Paulista de Medicina

Correspondência:

Kyung Koo Han

R. Raul Pompéia, 199, Apto 113 - Pompéia

05025-010 - São Paulo - SP

Tel: (11) 3862-8177

e-mail: kkh@ig.com.br

A isoflavona utilizada foi doada pela indústria farmacêutica Eugenbio CO., LTD. (Seoul, Coréia do Sul)

Conflito de interesses: Não mencionado. cida há algumas décadas e, por esta razão, são denominadas de fitoestrogênios. Historicamente, estas plantas foram utilizadas por povos das civilizações antigas. O pomegranato (Punica granatum) era o símbolo de imortalidade, fertilidade e amor na religião Oriental; na mitologia grega e judaica esta substância apresentava poderes lendários ${ }^{1}$. No Antigo Egito, o pólen do grão da planta Phonix dactylifera era usado para induzir a fertilidade nas mulheres.

O aparecimento da soja remonta há mais de cinco milênios na região da Coréia e Manchúria. Hoje, no sudeste asiático, a soja re- 
presenta 20 a $60 \%$ da proteína ingerida pela população, na forma de queijo de soja (Dubu), sopa de soja, pasta de soja fermentada (Doenjang), leite de soja $(D u y u)$ e extrato de soja ${ }^{2}$. No Ocidente, com o desenvolvimento da indústria, e conseqüente mudança dos hábitos alimentares e aumento da ingestão de gordura animal, houve elevação da incidência das doenças cardiovasculares, que começaram a se destacar como preocupação na área de saúde pública. Hoje, esta doença representa a primeira causa de mortalidade nos EUA ${ }^{3}$.

Os três maiores grupos de fitoestrogênios encontrados são: flavonas, isoflavonas e cumestranos. O poder estrogênico destas substâncias é variável. O grupo de isoflavona (malonilgenistina, malonildaidzina, genistina, daidzina, genisteína, daidzeína, acetildaidzina, gliciteína, acetilgenistina e equol) tem maior atividade estrogênica e maior afinidade pelos receptores ${ }^{4-9}$. Um dos metoxi-derivados da isoflavona, chamado de biochanin A, não se liga aos receptores de estrogênios mas tem efeito estrogênico in vivo. A daidzeína e a formonetina têm maior afinidade pelos receptores estrínicos do que os metoxi-derivados, mas ambos possuem efeito estrogênico fraco in vivo ${ }^{7,8}$. A metilação poderia ser o mecanismo pelo qual o efeito estrogênico da isoflavona é reduzido. A diferença existente entre a genisteína e a daidzeína se deve à presença do grupo 5-hidroxila de genisteína ${ }^{3-5}$.

As propriedades estrogênicas e antiestrogênicas dos fitoestrogênios decorrem da sua interação com os receptores de estrogênios ${ }^{4-6}$. Os lignanos e as isoflavonas têm atividade estrogênica fraca e, às vezes, antiestrogênica (como o tamoxifeno). Os flavonóides apresentariam atividade antiviral, anticarcinogênica, bactericida, antifúngica, antioxidante, antimutagênica, antihipertensiva, antinflamatória e antiproliferativa, como foi relatado em alguns experimentos ${ }^{4,5,7}$. Estudos recentes têm assinalado efeitos benéficos dos fitoestrogênios na prevenção de várias doenças crônicas como os cânceres de cólon, mama e próstata, e as doenças cardiovasculares. Nas pacientes na pós-menopausa, observou-se que são capazes de reduzir os sintomas e que poderiam prevenir algumas doenças crônicas que ocorrem no climatério, como a osteoporose $e^{3,10-12}$.

Acredita-se que os fitoestrogênios produzem alguns dos efeitos do estrogênio, porém, não seriam carcinogênicos ${ }^{9}$. A genisteína funcionaria como potente inibidor da oncogênese ${ }^{13,14}$, pois inibe a tirosina quinase (PTK), o fator de crescimento epidérmico (EGF-R), as DNA topoisomerases I e II, a quinase ribossômica S6, assim como a angiogênese e a diferenciação celular in vivo. Além disso, inibiria a produção de radicais livres, modula- ria o ciclo celular e, eventualmente, poderia precipitar a apoptose ${ }^{13}$.

O efeito estrogênico dos fitoestrogênios é mais facilmente observado nas mulheres do sudeste asiático, onde o consumo de isoflavona, presente na soja, é 20 vezes maior do que na população dos EUA, que consume mais produtos industrializados à base de carne vermelha ${ }^{5}$.

Os benefícios que os fitoestrogênios proporcionam ao sistema cardiovascular já foram referidos por diversos autores ${ }^{15-18}$. Segundo Terpstra e Beynen $^{19}$, Sirtoli e Lovati ${ }^{20}$, os fitoestrogênios reduzem os níveis de LDL e a taxa total de colesterol sangüineo tanto em animais quanto em humanos. Em relação a esses achados, destaca-se que a incidência de coronariopatias na população asiática, comparada com a população dos EUA, é dez vezes menor ${ }^{21}$.

Com o aumento da expectativa de vida das mulheres e, ao mesmo tempo, com o crescimento dos fatores que agravam as doenças crônicas, como fumo, estresse, vida sedentária e hábito alimentar rico em gordura, entre outros, a terapia de reposição hormonal nas mulheres na pós-menopausa tem sido cada vez mais indicada. A isoflavona poderia ser alternativa terapêutica neste período da vida da mulher. O consumo diário de 45 a 100 $\mathrm{mg}$ de isoflavona, que equivale a 60-100 g de soja, seria suficiente para se obter benefícios ${ }^{22-24}$, em especial para abolir os sintomas vasomotores.

O objetivo do presente estudo é avaliar a ação da isoflavona no alívio dos sintomas climatéricos e sobre alguns parâmetros laboratoriais, como os níveis de colesterol total e suas frações, bem como os de triglicérides.

\section{Pacientes e Métodos}

O grupo de estudo foi constituído pelas pacientes assistidas no Setor de Climatério do Departamento de Ginecologia da Universidade Federal de São Paulo - Escola Paulista de Medicina (UNIFESP-EPM), no período de agosto a novembro de 1999. O diagnóstico de menopausa foi baseado nos dados clínicos, ou seja, amenorréia de pelo menos 12 meses, confirmado pela elevação da gonadotrofina folículo-estimulante ( $\mathrm{FSH}>35 \mathrm{mUI} /$ $\mathrm{mL})$ e baixos valores de estrogênio $\left(\mathrm{E}_{2}<20 \mathrm{ng} / \mathrm{dL}\right)$. As pacientes que preencheram este critério, e que não estavam em tratamento hormonal prévio e não fizeram uso de herbais ou dieta rica em alimentos à base de soja foram selecionadas para entrar neste estudo. Assim, estudamos 80 pacientes em estudo duplo-cego randomizado, com duração de 16 semanas. Todas as pacientes foram 
orientadas sobre possiveis efeitos colaterais e concordaram em participar deste estudo por meio de consentimento escrito em documento formulado e aprovado pelo Comitê de Ética em Pesquisa da UNIFESP-EPM. As pacientes foram divididas em dois grupos de 40 patientes: GI (isoflavona) que foi tratado com $100 \mathrm{mg}$ de isoflavona, divididos em 3 tomadas diárias (cada cápsula com 33,3 mg de isoflavona); GCtr (placebo) recebeu composto de glicose com a proteína da soja.

Os sintomas foram avaliados usando-se o indice menopausal de Kupperman antes e após o tratamento. As pacientes foram questionadas verbalmente durante as consultas sobre os itens deste índice, como fogachos, insônia, mialgia ou artralgia, formigamento, palpitação, parestesia, nervosismo, tontura, cefaléia e melancolia. Para a pontuação dos fogachos, determinamos que o sintoma leve teria freqüência menor 5 por dia; moderado entre 5 e 10 por dia, ao passo que os casos acentuados, mais que 10 fogachos em um dia. Os outros sintomas obedeceram aos critérios descritos por Kupperman. Foi analisada a somatória de todos os sintomas do índice menopausal para fins de comparação entre os grupos. Todas as pacientes foram consultadas pelo mesmo examinador no início e no final da pesquisa para atenuar erros de pontuação; entretanto, este médico não tinha informações sobre o tratamento de cada paciente para não comprometer o estudo duplo-cego.

As pacientes foram submetidas à coleta de sangue para dosagens bioquímicas de triglicérides, colesterol total e frações, estradiol e FSH. Além disso, foram submetidas à avaliação ultrasonográfica, no início e no final deste trabalho. O protocolo incluiu também o rastreamento de câncer ginecológico por meio da citologia oncológica cervicovaginal, mamografia bilateral e ultra-som transvaginal.

Nosso protocolo de pesquisa seguiu os seguintes passos: todas as pacientes ao submeterem ao estudo, realizaram os exames de rotina para prevenção de câncer ginecológico (citologia cervicovaginal, mamografia e ultra-sonografia transvaginal), cujos resultados não serão discutidos neste trabalho. Após o exame físico, que foi efetuado em intervalos de 4 semanas, registraram-se os valores do peso e da altura e calculouse o índice de massa corpórea (IMC) antes e após o tratamento.

Em resumo, a análise descritiva das variáveis de interesse foi realizada usando-se os dados do índice de Kupperman, os níveis séricos de FSH, estradiol, colesterol, além da espessura endometrial e do IMC, que serão os parâmetros apresentados e discutidos neste trabalho. Para estas vari- áveis foram calculadas algumas medidas resumo como média e erro-padrão ${ }^{25}$. A análise do índice de Kupperman foi feita usando o teste não paramétrico de Kruskal-Wallis e o pós-teste de múltiplas comparações de Dunn. A comparação dos demais dados colhidos dos dois grupos estudados (isoflavona e placebo) foi realizada pelo teste $t$ para duas amostras independentes ${ }^{25}$. Já para a análise dos dados no mesmo grupo (isoflavona ou placebo), comparando-se os dados de antes e após o tratamento, usou-se o teste $t$ pareado $^{25}$. O nível de significância foi fixado em $5 \%$.

\section{Resultados}

Após a pontuação dos sintomas climatéricos avaliados pelo índice de Kupperman, 32 (80\%) das pacientes do GI (isoflavona) referiram melhora, com redução de aproximadamente $60 \%$ na pontuação antes do início do tratamento, ao passo que no GCrt (controle) apenas $5(12,5 \%)$ das pacientes referiram melhora. Destas últimas, a porcentagem de melhora foi de aproximadamente $40 \%$. A Tabela 1 mostra as médias e os erros-padrão dos valores calculados do índice de Kupperman. Observa-se que houve, no GI (isoflavona) queda significante após o tratamento; tal fato não foi verificado no GCtr (controle). O principal item do índice menopausal que diminuiu foi o número de fogachos das pacientes do GI (isoflavona).

Tabela 1 - Índice de Kupperman das pacientes antes e após o tratamento com isoflavona em comparação às que receberam placebo.

\begin{tabular}{lcccc}
\hline & \multicolumn{2}{c}{ GI (Isoflavona) } & \multicolumn{2}{c}{ G2 (Placebo) } \\
& Antes & Depois & Antes & Depois \\
\hline Média & 44,6 & $24,9^{*}$ & 40,3 & 41,6 \\
Erro & 1,0 & 1,7 & 1,2 & 1,1 \\
Padrão & 1,0 & 1,7 & 1,2 & 1,1 \\
\hline
\end{tabular}

* $p<0.001$ (teste não paramétrico de Kruskal-Wallis).

Pós-teste de Dunn para múltiplas comparações:

${ }^{*} p<0.001$ quando se compara GI depois ao GI antes, GCtr antes e GCTr depois. Não há diferenças estatísticas nas outras comparações

Os níveis de FSH não apresentaram modificações significantes, quando se compararam os dados das pacientes do GI $(667,1 \pm 3,2)$ em relação ao GCtr $(65,4 \pm 3,7)$ no final do tratamento. Entretanto, os valores de estradiol foram maiores no GI $(19 \pm 2,2)$ em comparação aos do GCtr $(10,9 \pm 0,6)$ após o tratamento.

Das 35 pacientes do GI (isoflavona) que apresentaram níveis séricos anormais de colesterol (superiores a $200 \mathrm{mg} / \mathrm{dL}$ ), houve diminuição em 
$30(85,7 \%)$ pacientes, ao passo que no GCtr (controle) a diminuição ocorreu em apenas $13(34,2 \%)$ das 38 pacientes que apresentaram alteração plasmática prévia. A Tabela 2 mostra os valores médios e os erros padrões dos níveis séricos de colesterol total das pacientes de ambos os grupos.

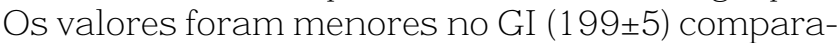
tivamente aos do GCtr $(225,6 \pm 6,2)$.

Tabela 2 - Índice de colesterol das pacientes antes e após o tratamento com isoflavona, em comparação com as que receberam placebo

\begin{tabular}{lccrr}
\hline & \multicolumn{2}{c}{ GI (Isoflavona) } & \multicolumn{2}{c}{ GCTR (Placebo) } \\
& Antes & Depois & Antes & Depois \\
\hline Média & 266,6 & $199^{*}$ & 226,8 & 225,6 \\
Erro-Padrão & 7,7 & 5,0 & 8,0 & 6,2 \\
\hline
\end{tabular}

Teste $t$ para 2 amostras independentes

GI vs GCTR (antes) : $p=0,99$, ou seja, > $5 \%$ GI= GCTR

GI vs GCTR (depois): $p=0,0013$, ou seja, <5\% Gl< GCTR*

Teste $t$ pareado

*p<0.05 comparando-se os valores antes e após tratamento no GI.

A ultra-sonografia transvaginal mostrou que apenas $4(1 \%)$ pacientes do GI apresentaram valores acima de $4 \mathrm{~mm}$, ao passo que no GCtr, observou-se isto em apenas $2(0,5 \%)$ casos, não havendo diferença significante entre os dois grupos estudados.

Em 18 pacientes do GI, 45\% sofreram redução e 3 apresentaram aumento do IMC. Já no GCtr, só duas pacientes sofreram redução do IMC e 12 pacientes apresentaram aumento. As demais pacientes de ambos os grupos não mostraram alteração significante do IMC. Na Tabela 3 foram colocados as médias dos valores do IMC de ambos os grupos antes e após o tratamento. Não houve diferenças estatísticas entre os grupos.

Tabela 3 - Variação do índice de massa corpórea (IMC) das pacientes antes e após 0 tratamento com isoflavona, em comparação às que receberam placebo

\begin{tabular}{lcccc}
\hline & \multicolumn{2}{c}{ GI (Isoflavona) } & \multicolumn{2}{c}{ GCTR (Placebo) } \\
& Antes & Depois & Antes & Depois \\
\hline Média & 25,60 & 25,01 & 24,19 & 24,43 \\
Erro-Padrão & 0,57 & 0,55 & 0,53 & 0,53 \\
\hline
\end{tabular}

Teste $t$ para 2 amostras independentes e pareado: não houve diferenças estatísticas.

\section{Discussão}

Acredita-se que a isoflavona mimetize algumas das ações dos estrogênios naturais, pois, os fitoestrogênios atuaram nos receptores de estro- gênio; assim, melhoraria os sintomas indesejáveis da síndrome do climatério. Do mesmo modo que o estrogênio natural, os fitoestrogênios agiriam nos centros termorreguladores de hipotálamo, atenuando os sintomas vasomotores, como observou-se no presente trabalho. Tais resultados são semelhantes aos encontrados na literatura, que relacionaram o uso de isoflavona com a melhora deste sintoma ${ }^{3,9,12,17,26}$. Em outro trabalho realizado com isoflavona, constatou-se que ocorre redução importante dos números dos fogachos das pacientes comparando-se ao placebo ${ }^{26}$. Entretanto, faltam trabalhos consistentes na literatura que demonstrem que os efeitos da isoflavona sejam semelhantes aos do estrogênio.

Estes dados também estão de acordo com os da literatura, pois, demonstraram que a ingestão de soja e seus derivados ou as isoflavonas não modificaram os níveis de gonadotrofinas ${ }^{3,8,17}$. Apesar da elevação dos níveis de estrogênio após o tratamento não serem importantes para justificar a melhora clínica destas pacientes, não podemos considerar que esse achado possa ter influenciado parcialmente nesses resultados. Esse achado foi inesperado e poderia ser explicado pela eventual interação da isoflavona com a proteína carreadora dos esteroíde sexuais ${ }^{7,12}$.

A diminuição dos níveis séricos de colesterol era esperada e também foi verificada em outros estudos ${ }^{12-18}$, especialmente nos países do Oriente, onde o consumo de soja e seus derivados é aproximadamente 20 vezes superior ao verificado nos Estados Unidos da América. É importante ressaltar que neste país ocorrem também mais mortes decorrentes de doenças cardiovasculares do que nos países do Oriente ${ }^{16}$. A redução dos níveis de colesterol plasmático é explicada parcialmente pela ação da isoflavona no metabolismo hepático e também na sua ação sobre a globulina carreadora do hormônio esteróide ${ }^{7}$.

Quanto à redução do peso corporal das pacientes que usaram isoflavona, como este fenômeno é multifatorial, a ingestão de isoflavona isoladamente não seria o único fator responsável pela sua diminuição. Este achado também foi registrado em outros trabalhos ${ }^{6-8}$.

Com relação à espessura do endométrio, não detectamos variações significativas antes e após tratamento com isoflavona. Assim, no período de tempo avaliado (16 semanas) não houve estímulo endometrial.

Portanto, baseado nos dados de nosso estudo, a isoflavona constitui alternativa para atenuar os sintomas climatéricos de pacientes com risco maior de desenvolverem câncer ginecológico e para as que possuem contra-indicação para o uso de estrogênio. 


\section{ABSTRACT}

Purpose: to evaluate the estrogenic effects of soy phytoestrogens in postmenopausal patients.

Method: we selected postmenopausal patients from the Climacteric Service of the Gynecology Department of the Federal University of São Paulo - Escola Paulista de Medicina. The patients were divided into two groups: GI $(\mathrm{n}=40)$ received daily $100 \mathrm{mg}$ isoflavone (3 x $33.3 \mathrm{mg} /$ day); and GCtr $(n=40)$ received daily placebo. We included in this study the Kuppermann menopausal index, physical examination and the biochemical determination of total and fractions of cholesterol, triglycerides, estradiol, FSH e LH. We also performed sonographic examination to measure endometrial thickness. To compare baseline and after treatment values of both groups, the nonparametric KruskalWallis test was used for the Kuppermann index, while the $\mathrm{t}$ test was performed for other data.

Results: evaluation of the Kupperman Menopausal Index, $80 \%$ patients of GI showed a decrease in the total score, while the improvement of symptoms in GCtr was 12,5\%. The analysis of total cholesterol levels showed that 35 patients of GI had levels above $200 \mathrm{mg} / \mathrm{dL}$, and only 13 of GCtr presented similar levels. The body mass index reduction occurred in GI and the control group showed no changes. We also verified that echo endometrial thickness and FSH levels were similar to baseline values. However the values of estrogen increased after isoflavone treatment.

Conclusion: we concluded that $100 \mathrm{mg} /$ day isoflavone may be an alternative treatment for attenuating the climacteric symptoms in $80 \%$ of isoflavone users and that it reduced total cholesterol.

KEY WORD: Phytoestrogen. Menopause. Climacterium. Kupperman index.

\section{Agradecimentos}

Agradecemos aos Srs. José Marcos G. Mandarino e Akio Kikuchi (EMBRAPA-SOJA) e Dr. Tae Wan Kwon e Dra. Sung Ran Kim (Korea Food Research Institute) pelo inestimável esforço e colaboração na análise do isoflavona e pela colaboração com o nosso estudo

\section{Referências}

1. Fransworth NR, Bingel AS, Cordell GA, Crane FA, Fong HS. Potential value of plants as sources of new antifertility agents II. J Pharm Sci 1975; 64:717-54.
2. Kwon T W, Song Y S, Kim J S, Moon G S, Kim J I, Hong $\mathrm{J}$ H. Current research on the bioactive functions of soyfoods in Korea. Korea Soybean Digest 1998; 15:1-12.

3. Clarkson TB, Anthony MS, Hughes CL. Estrogenic soybean isoflavones and chronic disease. Risks and benefits. Trends Endocrinol Metab 1995; 6:11-6.

4. Kondo H, Nakajima N, Yamamoto N, et al. BE-14384 substances, new specific estrogen- receptor binding inhibitors. Production, isolation, structure determination and biological properties. J Antibiot (Tokyo) 1990; 43:1533-42.

5. Martin PM, Horwitz KB, Ryan DS, McGuire WL. Phytoestrogen interaction with estrogen receptors in human breast cancer cells. Endocrinology 1978; 103:1860-7.

6. Setchell KD, Gosselin SJ, Welsh MB, et al. Dietary estrogens - a probable cause of infertility and liver disease in captive cheetahs. Gastroenterology 1987; 93:225-33.

7. Knight DC, Eden JA. A review of the clinical effects of phytoestrogens. Obstet Gynecol 1996; 87:897-904.

8. Kim S R, Kim S D. Studies on soybean isoflavones. J Agric Sci 1996; 38:155-65.

9. Shemesh M, Lindner HR, Ayalon N. Affinity of rabbit uterine oestradiol receptor for phyto-oestrogens and its use in a competitive protein-binding radioassay for plasma coumestrol. J Reprod Fertil 1972; 29:1-9.

10.Gennari C, Adami S, Agnusdei D, et al.. Effect of chronic treatment with ipriflavone in postmenopausal women with low bone mass. Calcif Tissue Int 1997; 61 (Suppl 1):S19-22.

11.Arjmandi BH, Alekel L, Hollis BW, et al. Dietary soybean protein prevents bone loss in an ovariectomized rat model of osteoporosis. J Nutr 1996; 126:161-7.

12.Agnusdei D, Bufalino L. Efficacy of ipriflavone in established osteoporosis and long-term safety. Calcif Tissue Int 1997; 61 (Suppl 1):S23-7.

13.Barnes S. Effect of genistein on in vitro and in vivo models of cancer. J Nutr 1995; 125 Suppl:777S$783 S$.

14.Peterson G. Evaluation of the biochemical targets of genistein in tumor cells. J Nutr 1995; 125 Suppl:784S-789S.

15.Bakhit RM, Klein BP, Essex-Sorlie D, Ham JO, Erdman JW Jr, Potter SM. Intake of $25 \mathrm{~g}$ of soybean protein with or without soybean fiber alters plasma lipids in men with elevated cholesterol concentrations. J Nutr 1994; 124:213-22.

16.Ni W, Tsuda Y, Sakono M, Imaizumi K. Dietary soy protein isolate, compared with casein, reduces atherosclerotic lesion area in apolipoprotein Edeficient mice. J Nutr 1998; 128:1884-9. 
17. Nestel PJ, Yamashita T, Sasahara T, et al. Soy isoflavones improve systemic arterial compliance but not plasma lipids in menopausal and perimenopausal women. Arterioscler Thromb Vasc Biol 1997; 17:3392-8.

18. Nagata C, Takatsuka N, Kurisu Y, Shimizu H. Decreased serum total cholesterol concentration is associated with high intake of soy products in Japanese men and women. J Nutr 1997; 128:20913.

19. Terpstra AH, Beynen AC. Density profile and cholesterol concentration of serum lipoproteins in experimental animals and human subjects on hypercholesterolaemic diets. Comp Biochem Physiol B 1984; 77:523-8.

20.Sirtori CR, Lovati MR. Soy proteins and cardiovascular disease. Curr Atheroscler Rep 2001; 3:47-53.

21.Clarkson TB, Anthony MS, Williams JK, Honore EK, Cline JM. The potential of soybean phytoestrogens for postmenopausal hormone replacement therapy. Proc Soc Exp Biol Med 1998; 217:365-8.
22-Fukutake M, Takahashi M, Ishida K, Kawamura H, Sugimura T, Wakabayashi K. Quantification of genistein and genistin in soybeans and soybean products. Food Chem Toxicol 1996; 34:457-61.

23.Anderson JJB, Garner SC. The effects of phytoestrogens on bone. Nutr Res 1997; 17:161732.

24.Baird DD, Umbach DM, Lansdell L, Hughes CL, et al. Dietary intervention study to assess estrogenicity of dietary soy among postmenopausal women. J Clin Endocrinol Metab 1995; 80:1685-90.

25.Bussab WO, Morettin PA. Estatística básica: métodos quantitativos. $4^{\mathrm{a}}$ ed. São Paulo: Atual; 1987. p.321.

26.Han KK, Soares JM Jr, Haidar MA, de Lima GR, Baracat EC. Benefits of soy isoflavone therapeutic regimen on menopausal symptoms.Obstet Gynecol 2002; 99:389-94.

Recebido em: 25/7/2000 Aceito com modificações em: 27/9/2002 